\title{
Differences in resource allocation to stolon branches of Kopu white clover genotypes induced by manipulation of rooting
}

\author{
M. LÖTSCHER and M.J.M. HAY \\ AgResearch Grasslands, Private Bag 11008, Palmerston North
}

\begin{abstract}
White clover (Trifolium repens L.) genotypes of the cv. Grasslands Kopu which had differing numbers of vascular bundles within their stolons were tested for potential for resource sharing between shoot branches on either side of parent axis. Genotypes with a high (10-13) number of vascular bundles (H-genotypes) had larger leaves, thicker stolons but lower node appearance rates than genotypes with a low (8-9) number of vascular bundles (L-genotypes). In the first experiment all roots on one side of the parent axis were severed two days before plants were labelled with ${ }^{32} \mathrm{P}$ (uptake period $24 \mathrm{~h}$ ). In H-genotypes, distribution of radioactive phosphorus $\left({ }^{32} \mathrm{P}\right)$ exported from a nodal root was restricted largely to near-side branches, i.e. branches which were on the same side of the parent axis as the source root, with only $4 \%$ allocated to far-side branches. In contrast about $20 \%$ of ${ }^{32} \mathrm{P}$ exported from the source root was transported to far-side branches in L-genotypes. In the second experiment, where all far-side roots were severed three weeks before harvest, growth of far-side branches was similarly reduced in L-genotypes and $\mathrm{H}$ genotypes. When root formation along one side of the parent axis was prevented during the entire experimental period the mean ratio of total dry weight between nearand far-side branches was higher in H-genotypes compared to L-genotypes.
\end{abstract}

Keywords: branch development, nutrient distribution, phosphorus, vascular bundle, white clover, Trifolium repens $\mathrm{L}$.

\section{Introduction}

White clover (Trifolium repens L.) is a plagiotropic species that persists in moist, temperate environments via clonal growth. Each node has two root primordia and these confer each node with potential to tap a new site of mineral resources. However, under field conditions roots often fail to develop or die in early stages of development (Chapman 1983; Newton \& Hay 1994). Hence plants may have non-rooted sections of stolon where leaves, branches or flowers are dependent on the distribution of resources from nodal roots located on nodes distanced from them. It has been shown that in large-leaved genotypes, resources $\left({ }^{32} \mathrm{P}\right)$ exported from a nodal root were mainly transported to near-side branches, i.e. branches which were on the same side of the parent axis as the source root (Hoshino 1974; Hay \& Sackville Hamilton 1996). In these genotypes distribution of mineral nutrients was consistent with the vascular system of the stolon as traces directly connecting the axial bundles on one side with those on the other side of the stolon have not been reported. However in a smallleaved genotype where plants had a few basal roots and a root (source root) $12-15$ nodes distal to them, ${ }^{32} \mathrm{P}$ exported from the source root was transported to branches on both sides of the parent axis (Kemball \& Marshall 1994). The higher resource sharing between near- and far-side branches reported by Kemball and Marshall (1994) could have been either an effect of genotypic variability or an increase in lateral transport (defined here as transport from axial bundles on one side of the stolon to axial bundles on the other side) resulting from an altered source-sink balance within the plant induced by the rooting pattern. It has been shown that phyllotaxic constraints on the pattern of nutrient distribution can be removed when the source-sink balance of the plant is modified (Wardlaw 1990).

In a preliminary experiment we found that resource sharing (increased lateral transport of ${ }^{32} \mathrm{P}$ and ${ }^{45} \mathrm{Ca}$ ) did not occur in a large-leaved genotype even when all farside roots were severed two days before the plants were supplied with radioisotopes. In contrast, a small-leaved genotype showed little resource sharing when nonstressed but a marked increase in resource sharing when far-side roots were severed. These two genotypes differed in many morphological characteristics but also in the number of vascular bundles within their stolons. In order to minimise possible confounding effects of contrasting morphology, observations were made of genotypes selected within a single large-leaved cultivar (Grasslands Kopu) for high (10-13) and low (8-9) vascular bundle number within stolons. The first experiment used genotypes with high (H-genotypes) and low (L-genotypes) numbers of vascular bundles to test whether differences in the potential for lateral transport of ${ }^{32} \mathrm{P}$ were correlated with the number of vascular bundles of the genotypes. In the second experiment we tested whether genotypic differences in the short-term distribution of radioisotopes had 
implications for the development and growth of plants over an extended time period.

\section{Methods}

\section{Plant culture}

Two Kopu genotypes with high and two with low numbers of vascular bundles were used for the experiments. Stolon cuttings taken from stock plants were placed in seed trays filled with sand and put in a glasshouse for establishment. Plants were inoculated with a commercial Rhizobium strain (Tui Inoculant Peat, $\mathrm{NZ}$ ) one week after planting and watered twice a day with nutrient solution containing $2.5 \mathrm{mM} \mathrm{N}\left(\mathrm{NH}_{4} \mathrm{NO}_{3}\right)$, $1.0 \mathrm{mM} \mathrm{P}, 1.0 \mathrm{mM} \mathrm{K}, 1.25 \mathrm{mM} \mathrm{Ca}, 0.5 \mathrm{mM} \mathrm{Mg}, 1.0$ $\mathrm{mM} \mathrm{S}, 0.079 \mu \mathrm{M} \mathrm{Cu}, 2.06 \mu \mathrm{M} \mathrm{Mn}, 11.7 \mu \mathrm{M} \mathrm{B}, 0.05$ $\mu \mathrm{M}$ Mo, and $0.38 \mu \mathrm{M} \mathrm{Zn} \mathrm{(pH} \mathrm{6.0).}$

\section{Experiment 1}

After four weeks of establishment, plants were transplanted into containers $(60 \mathrm{~cm}$ long x $16 \mathrm{~cm}$ wide x $12 \mathrm{~cm}$ deep) filled with sand (particle size $>1 \mathrm{~mm}$ ) and transferred to a growth room. Conditions of the growth room (National Climate Laboratory, Hort Research, Palmerston North) were: $22 / 17^{\circ} \mathrm{C}$ day/night temperature, $400 \mu \mathrm{mol} \mathrm{m} \mathrm{m}^{-2} \mathrm{~s}^{-1}$ photosynthetic photon fluence rate, $12 \mathrm{~h}$ photoperiod and $70 \%$ relative humidity. Rooting on branches was prevented by inserting clear polythene sheets under branches. A stainless steel column ( $3 \mathrm{~cm}$ diameter, $16 \mathrm{~cm}$ long) was inserted into the growth containers just beyond the apical bud of the parent axis. The column was filled with sand and had a water-holding capacity of $20 \mathrm{ml}$. Further growth of the parent axis was directed over the column so that the root from a single node could grow into the column. This root was targeted for ${ }^{32} \mathrm{P}$ uptake and was designated the source root. Two days before the plants were labelled with ${ }^{32} \mathrm{P}$, all plants were pre-treated by the removal of all far-side roots along the parent axis. On the day of labelling the plants were nine weeks old, the parent axis had about 20 nodes and the root supplied with ${ }^{32} \mathrm{P}$ was at the ninth node counting back from the apex. The plants were supplied with $0.51 \mathrm{MBq}{ }^{32} \mathrm{P}$ by applying to the column $20 \mathrm{ml}$ of nutrient solution 'spiked' with carrier-free isotope using a syringe. Isotope was applied $3 \mathrm{~h}$ into photoperiod and plants were harvested after $24 \mathrm{~h}$.

At harvest, plants were dissected into root, parent axis and each individual branch. Plant material was dried at $80^{\circ} \mathrm{C}$ for $48 \mathrm{~h}$ and weighed into scintillation vials. Plant material was then ashed at $550^{\circ} \mathrm{C}$ for $6 \mathrm{~h}$ and the ash taken up in $2 \mathrm{ml} 0.5 \mathrm{M} \mathrm{HCl}$. Twenty millilitre of scintillation solution (1:2 mixture of Triton X100 and toluene with $0.6 \mathrm{~g}$ Omnifluor per $100 \mathrm{ml}$ ) was added to the vials and activity of ${ }^{32} \mathrm{P}$ determined on a scintillation counter (Model 1219, LKB Wallac, Finland).

\section{Experiment 2}

Plants were grown in the glasshouse. Rooting on branches was prevented as described in experiment 1 . Three rooting treatments, replicated four times, were applied to the parent axis of the plants. Control plants (C) had roots on all nodes of the parent axis. In the second treatment (RP), formation of far-side roots was prevented by inserting strips of polythene under the nodes. In the third treatment (RS), on another set of four plants per genotype all far-side roots were severed when plants were 11 weeks old so that plants had similar treatment as plants of experiment 1. At harvest plants were 14 weeks old. Plants were dissected into main axis, near- and far-side branches and roots. Plant material was dried at $80^{\circ} \mathrm{C}$ for $48 \mathrm{~h}$ and weighed.

Figure 1: Percentage of total exported ${ }^{32} \mathrm{P}$ allocated to branches of white clover genotypes that had a high vascular bundle number (open) or a low vascular bundle number (hatched) within their stolons. All roots on the far-side of the parent axis were severed two days before ${ }^{32} \mathrm{p}$ labelling. The uptake period of ${ }^{32} \mathrm{P}$ was $24 \mathrm{~h}$. Branches were numbered in ascending order towards the apex. The source root was at the parent node of branch 0 . Data are means of two genotypes with four replicates each +SE.

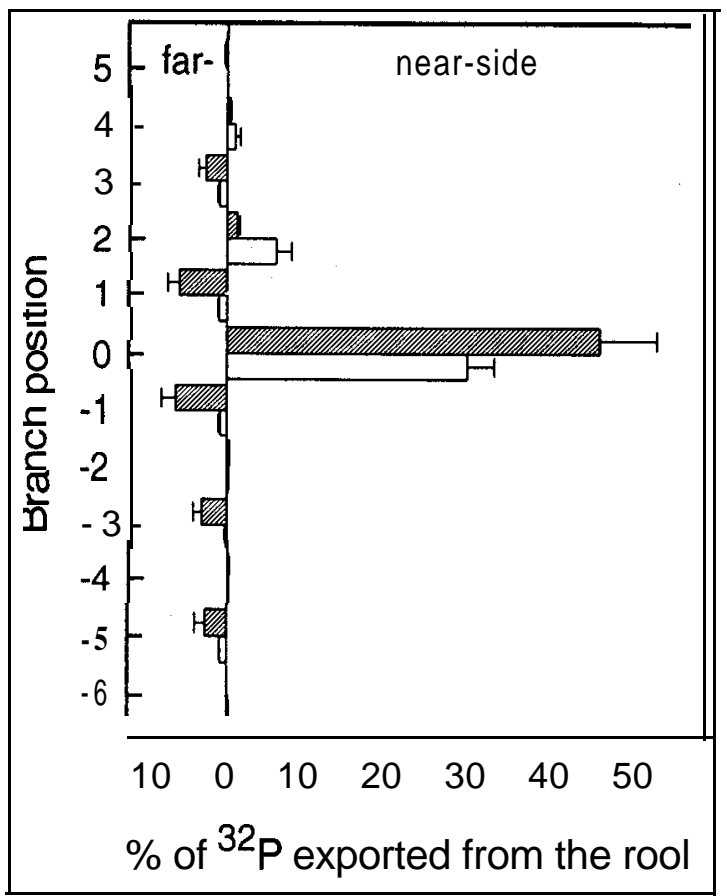




\section{Results and discussion}

In experiment 1 the allocation pattern of ${ }^{32} \mathrm{P}$ to branches differed significantly between genotypes with high $(\mathrm{H})$ and low (L) number of vascular bundles (Figure 1). In H-genotypes about $90 \%$ of the ${ }^{32} \mathrm{P}$ allocated to branches was in the distal, near-side group of branches, mainly in the branches at and two nodes distal to the source root. In contrast L-genotypes allocated significantly more ${ }^{32} \mathrm{P}$ to far-side branches than $\mathrm{H}$-genotypes. The patterns of intra-plant distribution of ${ }^{32} \mathrm{P}$ in the short term indicated that genotypic variation exists within white clover for the potential for lateral transport of nutrients across stolons. This variation was correlated with the number of vascular bundles within stolons of the genotypes. The very limited allocation of ${ }^{32} \mathrm{P}$ to far-side branches in H-genotypes suggested the possibility that in these genotypes growth and development would differ markedly between near- and far-side branches when far-side roots were absent on plants. By way of contrast the significant allocation of ${ }^{32} \mathrm{P}$ to far-side branches in L-genotypes suggested that differences in growth and development of near- and far-side branches should be less pronounced in these genotypes.

The second experiment was conducted over an extended period to examine if the genotypic differences that gave rise to differing ${ }^{32} \mathrm{P}$ allocations in the short term had longer-term implications for growth and development of branches on opposite sides of the parent axis. Unfortunately there were large variations in the dry weight of plants that could not be ascribed to treatment or block effects, which complicated the interpretation of the results. To remove the effects of variation in plant dry weight, ratios of total dry weight of near- and far-side branches were calculated (Table 1). For the treatment where roots were severed three weeks before harvest (RS) the dry weight ratios were higher than those of control plants but values for $\mathrm{H}$ - and L-genotypes were similar. The dry weight ratio differed even more from control plants when rooting on the far-side was prevented (RP) and there was also a difference between $\mathrm{H}$ - and L-genotypes. However, as indicated by the range of values for $\mathrm{H}$ genotypes (Table 1) there was great variability between individual plants of the H-genotypes subjected to the RP treatment. In some plants development of far-side branches was very limited whereas in others development was similar to that of L-genotypes. We conclude therefore that although this experiment provided some evidence indicating that the selected $\mathrm{H}$ - and L-genotypes differed in potential for resource sharing between near-
Table 1: Ratio of total dry weight between near- and far-side branches in genotypes of the white clover $\mathrm{cv}$. Grasslands Kopu with high $(\mathrm{H})$ and low $(\mathrm{L})$ number of vascular bundles within their stolons. All nodes of the parent axis were rooted $(\mathrm{C})$, roots on the far-side of the stolon were severed three weeks before harvest (RS), or root formation on the far-side of the parent axis was prevented during the entire experimental period (RP). Data are means of eight replicates. Figures in brackets are minimum and maximum values. Significance of differences between: genotypes, ns; Treatment, $\mathrm{P}<0.001$; Interaction, $\mathrm{P}<0.05$.

\begin{tabular}{lccr}
\hline Genotypes & C & RS & RP \\
\hline H & $1.2(0.7 ; 1.9)$ & $1.9(1.2 ; 2.5)$ & $5.7(2.8 ; 13.0)$ \\
L & $1.2(0.9 ; 1.5)$ & $1.9(1.1 ; 2.6)$ & $2.8(1.7 ; 3.8)$ \\
\hline
\end{tabular}

Plant characteristics of genotypes selected from the white clover cv. Grasslands Kopu for high (H) and (L) vascular bundle number within the stolon. Data are means of four replicates. Significance of differences between $\mathrm{H}$ - and $\mathrm{L}$-genotypes is presented.

\begin{tabular}{|c|c|c|c|c|c|}
\hline & \multicolumn{4}{|c|}{ - Genotypes - - } & \multirow{2}{*}{$\begin{array}{l}\text { Significance of } \\
\text { difference }\end{array}$} \\
\hline & $\mathrm{H} 1$ & $\mathrm{H} 2$ & L 1 & L 2 & \\
\hline No. of vascular bundles & $10-11$ & $12-13$ & $8-9$ & $8-9$ & \\
\hline (nodes day ${ }^{-1}$ ) & 0.24 & 0.23 & 0.26 & 0.25 & $P<0.001$ \\
\hline Mean leaf size $\left(\mathrm{cm}^{2}\right)$ & 15.6 & 11.2 & 6.4 & 6.6 & $P<0.001$ \\
\hline Mean internode length (mm) & 25.2 & 16.5 & 22.9 & 25.4 & ns \\
\hline Stolon diameter $(\mathrm{mm})$ & 3.2 & 3.0 & 2.6 & 2.4 & $P<0.01$ \\
\hline
\end{tabular}

and far-side branches, the results were by no means conclusive.

Genotypes selected for high and low vascular bundle number differed not only in potential for lateral transport of recently-assimilated ${ }^{32} \mathrm{P}$ but also in other physiological and morphological properties. H-genotypes had lower node appearance rates but produced larger leaves and thicker stolons than L-genotypes (Table 2). The vascular bundle number is often greater in genotypes with thicker stolons (Thomas 1987) and thicker stolons are usually associated with larger leaves (Caradus et al. 1993). Hence genotypic differences in distribution of ${ }^{32} \mathrm{P}$ in the short term may not necessarily be a result of differences in vascular bundle number but could also be influenced by other morphological or physiological properties of the genotypes.

\section{References}

Caradus, J.R.; Hay, M.J.M.; Mackay, A.D.; Thomas, V.J.; Dunlop, J.; Lambert, M.G.; Hart, A.L.; Van den Bosch, J.; Wewala, S. 1993. Variation within 
white clover (Trifolium repens L.) for phenotypic plasticity of morphological and yield related characters, induced by phosphorus supply. New Phytologist 123: 175-184.

Chapman, D.F. 1983. Growth and demography of Trifolium repens stolons in grazed hill pastures. Journal of Applied Ecology 20: 597-608.

Hay, M.J.M.; Sackville Hamilton, N.R. 1996. Influence of xylem vascular architecture on the translocation of phosphorus from nodal roots in a genotype of Trifolium repens L. during undisturbed growth. New Phytologist (in press).

Hoshino, M. 1974. Translocation and accumulation of assimilates and phosphorus in Ladino clover. Bulletin of the National Grassland Research Institute 5: 35-84.
Kemball, W.D.; Marshall, C. 1994. The significance of nodal rooting in Trifolium repens $\mathrm{L} .{ }^{32} \mathrm{P}$ distribution and local growth responses. New Phytologist 127: 83-91.

Newton, P.C.D.; Hay, M.J.M. 1994. Patterns of nodal rooting in Trifolium repens (L.) and correlations with stages in the development of axillary buds. Grass and Forage Science 49: 270-276.

Thomas, R.G. 1987. The structure of the mature plant. pp. 1-29. In: Baker, M.J.; Williams, W.M. (eds). White clover. CAB International, Wallingford, U.K. Wardlaw, I.F. 1990. The control of carbon partitioning in plants. New Phytologist 116: 341-381. 